\title{
THE PATHOGENESIS OF BRONCHIAL CATARRH AND OF ACUTE AND CHRONIC BRONCHITIS
}

\author{
BY \\ S. ENGEL \\ From the Department of Anatomy, Royal College of Surgeons of England
}

(RECEIVED FOR PUBLICATION OCTOBER 10, 1957)

"Chronic bronchitis" has recently been discussed from many points of view, all of them important enough, but very little has been said about the aetiology and pathogenesis of the condition. Investigations into the late stages of the disease, clinically, radiologically, or anatomically, do not show the course of the affliction, and, important as it may be to investigate the bacteriology, the effect of antibiotics, or the social conditions, nothing of this kind will elucidate the pathogenetic progress. On the other hand, especially in view of the growing incidence and danger of chronic bronchitis and bronchial carcinoma, it is necessary to investigate in a more detailed way the much neglected field of the normal and morbid anatomy of the bronchi. An attempt is therefore made in this paper to explain the basic pathogenetic factors and not to deal with acute exacerbations and their treatment.

\section{The Normal Bronchus}

The general structure of the bronchial tree has recently attracted much attention, particularly the "segmental" structure of the lung. This aspect of pulmonary anatomy will not, however, be dealt with in this paper, which is mainly concerned with the structure of the bronchial wall.

Fig. 1 shows the elements of which the bronchial wall is composed. (The numbering 1-6 is from within outwards.)

(1) The ciliated epithelium is, for the sake of simplicity, represented as a row of oblong, rectangular spaces. The columnar cells are rather spindle-shaped and arranged in two or three layers.

(2) The basal cells form a continuous row of small round cells. They rest on the basement membrane; they are germinal cells and serve as replacements for columnar cells.

(3) The basement membrane is thick or thin according to the bronchial calibre; it is a condensation of the tunica propria tissue (4) and serves as a foothold for the epithelium. The basal is cells (3) stick tightly to the membrane.

(4) The tunica propria is a thin layer of smooth connective tissue, rich in vessels; it contains also the elastic fibres. The elements numbered $1,2, \vec{c}$ 3 , and 4 together form the mucous membrane.

(5) The muscle is arranged in spirals rather than in circles; it acts as the motor of the mucous of membrane.

(6) The tunica fibro-cartilaginea consists of strong fibrous and elastic strands and is reinforced by cartilage, decreasing in strength towards the periphery. This layer gives the bronchus its stability and elasticity (Engel, 1947).

\section{The Mucous Membrane}

The membrane is normally arranged in a continuous row of folds (Fig. 2). Fig. 2 represents part of a $2.5 \mathrm{~mm}$. bronchus (internal diameter,

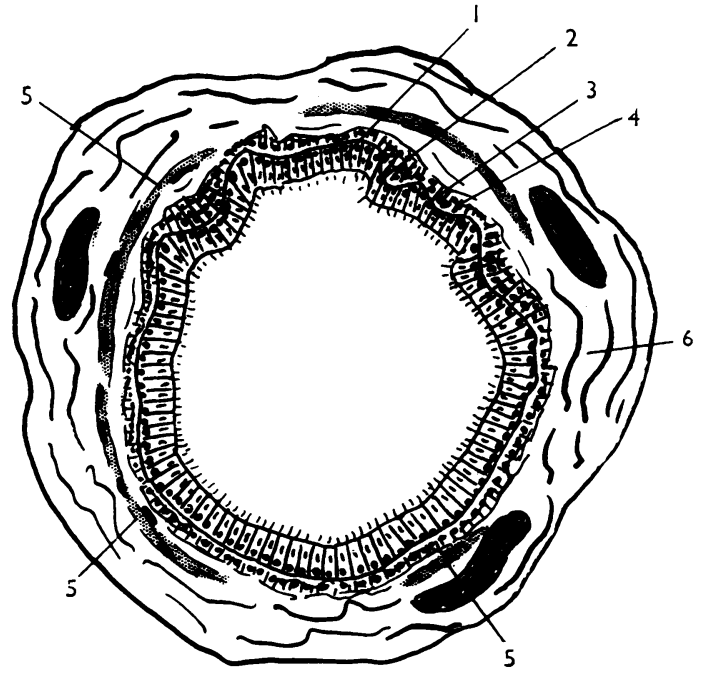

FIG. 1.-Diagrammatic representation of a cross-cut bronchus, . 35. 1 - ciliated epithelium; 2 =row of round basal cells; $3=$ base $\Omega$ ment membrane; 4 -tunica propria - elastica; $5=$ muscle 6 =tunica fibro-cartilaginea. 
measured from base to base of epithelium, $\times 60$ ). The folds are lined by ciliated columnar epithelium and their core consists of tunica propria with elastica. This arrangement used to and may still cause the illusion that the elastica of the bronchi runs in longitudinal strands. This is not so. Both the tunica propria and the elastica surround the bronchus evenly, provided that there are no folds, as can be seen in Fig. 1. In this case the mucosa has only a few shallow folds, and it is quite clear that they contain an excess of tunica propria tissue as compared with the smooth parts of the wall. The folds play an important part in the function and pathology of the bronchus.

\section{Bronchial Catarrh}

Bronchial catarrh follows a mild irritation and is not true inflammation. As the name indicates, catarrh is a condition where excess of fluid is a prominent feature. Catarrh is an uncomplicated superficial condition which abates within a few days and is therefore rarely accessible to anatomical investigation. The present author has had the opportunity of studying catarrhal bronchi in the post-mortem room and of carrying out histological examinations. It was evident that, in accordance with clinical observations, the catarrh usually affects only the trachea and main bronchi and stops abruptly at the hilum. Cases of this kind should be diagnosed as "tracheo-bronchial catarrh" and not as bronchitis. There is an abundance of mucus, which is sometimes yellowish, in the lower parts of the trachea and in the main bronchi, and this secretion may derive from the glands, which are particularly numerous in the trachea and main bronchi, attached to the mucous membrane. The epithelium itself may also produce mucus by a dramatic change whereby nearly every columnar cell is turned into a goblet cell. No other changes are seen in uncomplicated

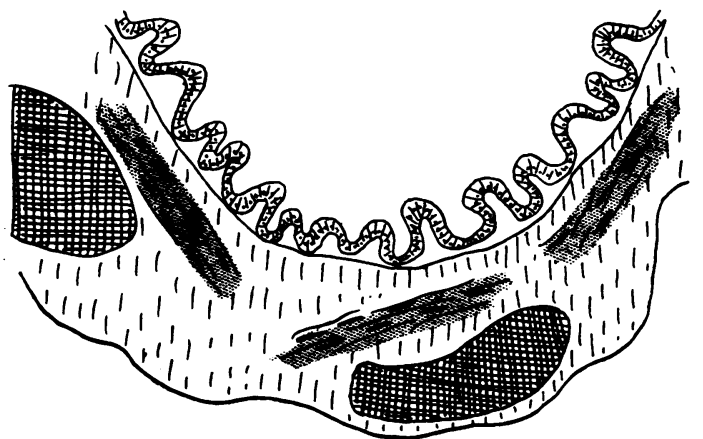

Fig. 2.-Part of a $2.5 \mathrm{~mm}$. bronchus to show the row of folds in which the mucous membrane is arranged, $\times 60$.

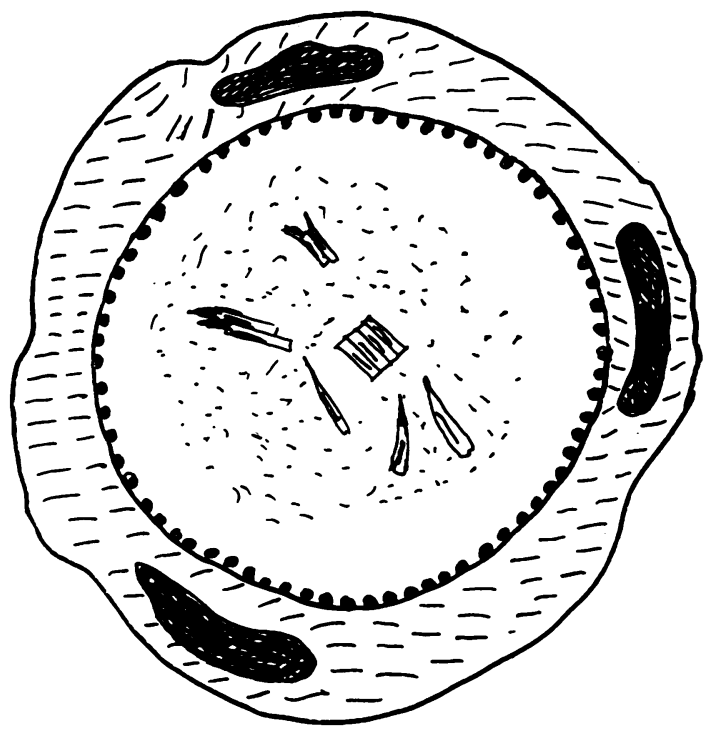

FIG. 3.-Slightly dilated bronchus, the columnar cells of which are cast off whereas the row of basal cells is intact. Mild acute bronchitis.

cases; there is no infiltration or destruction of mural elements and restitution to normality is the rule.

Bronchial catarrh occasionally occurs in medium and small bronchi. These tubes are less well provided with glands attached to the mucous membrane, and conversion of columnar cells into goblet cells is therefore the main source of mucus. This process can best be observed in cases of recent asthma where catarrh spreads to the small bronchi and bronchioli.

\section{Acute Bronchitis}

Acute bronchitis is chiefly localized in medium and small bronchi. The glands are hypertrophic and distended, columnar epithelium is often shed, and some of the dequamated cells may remain in the bronchial lumen mixed up with the secretion and may appear in the sputum (Fig. 3). A profuse quantity of mucus is produced and it may become purulent. The cells either keep their original shape or undergo degeneration and deformation. The basal cells, on the other hand, usually remain tightly adherent to the basement membrane ready for the renewal of lost columnar cells.

Potent infection is the usual cause of acute bronchitis, which may be a serious disease. The wall is deeply affected, generally infiltrated by lymphocytes, plasma cells, or even polymorphonuclears. Deeper layers are sometimes eroded, thus producing the picture of ulcerative bronchitis, 
but such destructive conditions are rare and are only mentioned to illustrate the many grades of bronchial inflammation. Severe or repeated attacks of bronchitis soften the bronchial wall with consequent, mostly cylindrical, dilatation. Bronchiectasis may be local or general, temporary or permanent, depending on many factors, such as the intensity of the inflammation or congenital weakness of the bronchi.

\section{Chronic Bronchitis}

Only one recent paper describes the morbid anatomical features of chronic bronchitis (Reid, 1954). Ogilvie and Newell (1957) refer only to Reid when discussing the pathology of chronic bronchitis. Reid is mainly concerned with late changes of the bronchioli rather than of the bronchi, arguing that obliterative bronchiolitis is the main cause of chronic bronchitis. She speaks of mild cases without describing the criteria of "mild" and her "advanced" cases obviously represent final stages with variegated secondary changes in the bronchioli and lung tissue. In mild cases hypertrophy of the mucus-secreting elements, glands and goblet cells, is noted. As conversion of columnar cells into goblet cells has been observed by the present author in catarrh, Reid's mild cases seem to be of catarrh rather than of bronchitis. There are of course no definite boundaries between catarrh and mild bronchitis, so that hypertrophy of goblet cells may also be a sign of mild bronchitis.

Pathogenetic Factors.-The condition affects mainly people of middle or old age. It is rampant in industrial areas, where the incidence and death rate are terrifying. Figures published from Leeds (Edwards, Buckley, Fear, Williamson, and Zinnemann, 1957) illustrate the severity of the problem. According to these authors the mortality in one year compared with that of pulmonary tuberculosis and bronchial carcinoma is as follows :

Pulmonary tuberculosis $\quad \ldots \quad \ldots \quad 67$ cases

$\begin{array}{llll}\text { Bronchial carcinoma } & \ldots & \ldots & 270 \text { cases }\end{array}$

Chronic bronchitis ... $\quad \ldots .4 . \ldots .463$ cases

Common experience shows that chronic bronchitis usually develops after repeated or protracted attacks of acute bronchitis; emphysema with rigidity of the dilated thorax (barrel chest) is often an additional if not the primary pathogenetic factor. How far atmospheric conditions or congenital susceptibility should be accused will not be discussed.

Repeated infections of the bronchial tree, which are so common a feature in chronic bronchitis, are not likely to be the cause of the disease, but rather they are conditioned by some change or susceptibility of the bronchi. Observations in early cases of repeated acute bronchitis suggest the following course. Repeated or protracted attacks of acute bronchitis, local or general, lead to slight cylindrical bronchiectasis, and this change is the forerunner of further attacks of bronchitis which will maintain or even increase the bronchial dilatation.

Slight cylindrical bronchiectasis produces important changes in the arrangement of the bronchial mucosa. As has been shown in Fig. 2, the mucous membrane is normally arranged in folds which serve a double purpose. It is evident that the membrane is larger than the internal bronchial $\dot{\omega}$ circumference. Therefore the folds permit the $N$ respiratory movements of the bronchi without 응 overstressing the membrane in the inspiratory widening of the bronchial lumen, and, secondly, $c$ they increase the surface of the membrane. The folded membrane is, as a simple matter of com- $\vec{\theta}$ mon sense, more efficient than a smooth lining for arresting and dealing with dust and bacteria suspended in the air. Bacilli caught in the folds are not only stopped there and expectorated, but in addition deprived of their virulence and/or destroyed. That this is so can be inferred from $\frac{0}{\circ}$ the fact that normal pulmonary tissue is practically $\stackrel{\varrho}{\Rightarrow}$ aseptic. This fact is the more startling as not all bacteria are arrested and expectorated, as can be concluded from the pathogenesis of primary tuberculosis. Therefore reduction of virulence is obviously the explanation of the fact that pul- $\frac{0}{8}$ monary tissue is aseptic in normal cases.

The folds are necessarily flattened in dilated bronchi, and the mucous membrane now adheres tightly to the bronchial wall. It might even be worse because the membrane will to some extent 윽 degenerate owing to the pressure in inspiration, $\rightarrow$ which normally is rendered ineffective because the folds give way by flattening. The antibacterial $N$ function of the mucosa is likely to be handicapped in dilated bronchi and acute exacerbations will 0 easily occur. The many attacks of acute bronchitis $\mathrm{W}$ in chronic cases are explicable in this way. A vicious circle will gradually develop, because each attack $\bullet$

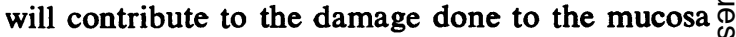
and will maintain or increase the bronchial dila- + tation. Finally the effect of chronic bronchitis $\frac{T}{0}$ affects the general condition of the subject, and $\stackrel{\odot}{\mathbb{D}}$ especially the heart, leading to the dangerous con- $\stackrel{?}{\mathcal{D}}$ dition of cor pulmonale. The secondary changes $\varnothing$ of chronic bronchitis which gradually lead to the fatal outcome are not the subject of the present 0 paper. 


\section{Conclusions and Summary}

Chronic bronchitis is not the result of repeated attacks of acute bronchitis ; they are not the cause of the disease, but are consequent on primary changes in the bronchial wall, especially in the mucous membrane. The condition begins with slight cylindrical bronchiectasis due to attacks of acute bronchitis or is a concomitant of emphysema. Dilatation of the bronchi leads to disarrangement and functional disorder of the mucous membrane. These changes pave the way for further attacks of acute bronchitis, which damage the mucosa, and bronchial dilatation. Eventually a vicious circle develops and the condition grows worse until the bronchial disintegration is irreparable and secondary changes in the bronchial tree and in the lung tissue complicate the situation, affect the general condition, and lead to cor pulmonale.

\section{REFERENCES}

Edwards, G., Buckley, A. R., Fear, E. C., Williamson, G. M., and Zinnemann, K. (1957). Brit. med. J., 2, 259.

Engel, S. (1947). The Child's Lung. Edward Arnold, London. Ogilvie, A. G., and Newell, D. J. (1957). Chronic Bronchitis in Newcastle-upon-Tyne. Livingstone, Edinburgh, and London.

Newcastle-upon-Tyne. Livingstone,
Reid, L. McA. (1954). Lancet, 1, 275. 